\title{
Relación entre la amplificación del oncogén C-erbB-2 y parámetros histológicos con sobrevida libre de enfermedad en pacientes con cáncer de mama
}

\author{
Alicia E. Rojas-Atencio' ${ }^{1}$, Ivonne Valbuena ${ }^{1}$, \\ Karelis Urdaneta ${ }^{1 \mathbf{a}}$, Marisol Soto-Quintana ${ }^{1 b}$, \\ Francisco Álvarez-Nava ${ }^{1}$, Leonardo González ${ }^{2}$, \\ María E. Viloria-Alvarado ${ }^{3}$, Rita Quintero-Bellagamba ${ }^{3 c}$ \\ Relationship of oncogene C-erbB-2 \\ amplification in breast cancer with \\ pathological parameters and disease \\ free survival
}

\begin{abstract}
region q21 and codifies a $185 \mathrm{Kd}$ protein, with tyrosine kinase activity. The amplification of this gene is associated with relapse and lower survival in breast cancer. Overexpression of this gene can be detected by immunohistochemistry (IHC). However, fluorescence in situ hybridization (FISH) and chromogenic in situ hybridization (CISH) allow the simultaneous analysis of morphology and overexpression of the gene. Aim: To evaluate the relationship of c-erbB-2 oncogene amplification measured by FISH with histological graduation, presence of positive Iymph nodes and evolution of breast cancer. Patients and methods: One hundred and ten tissue samples of invasive ductal or lobulillar breast cancer, positive for c-erbB-2 oncogene by IHC were analysed. The presence of c-erbB-2 oncogene amplification was subsequently analyzed by FISH. Results: There was a significant association of c-erbB-2 amplification by FISH with pathological graduation of the tumor, number of regional Iymph nodes involved and disease free survival. Conclusions: Proto-oncogene c-erbB-2 amplification is a good indicator of bad prognosis in invasive breast cancer (Rev Méd Chile 2005; 133: 151-7).
\end{abstract}

(Key Words : Brast neoplasms; Genes, erbB; Proto-oncogenes)

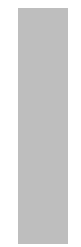

\begin{abstract}
Recibido el 2 de abril 2004. Aceptado en versión corregida el 14 de diciembre 2004.
Trabajo financiado parcialmente por CONDES-LUZ Proyecto №. CC 0287-01

${ }^{1}$ Unidad de Genética Médica. Facultad de Medicina, Universidad del Zulia. Maracaibo, Venezuela.

${ }^{2}$ Hospital Clínico. Servicio de Oncología. Maracaibo, Venezuela. ${ }^{3}$ Instituto de Investigaciones Biológicas, Facultad de Medicina, Universidad del Zulia-Venezuela

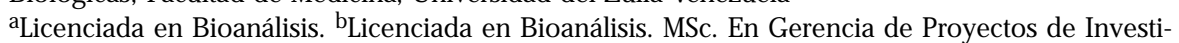
gación. 'Licenciada en Biología.
\end{abstract}

Correspondencia a: Dra. Alicia Rojas de Atencio, Unidad de Genética Médica, Hospital Universitario de Maracaibo, 2do Piso. Apartado Postal 15.210 oficina Galerías Maracaibo, Edo. Zulia. Venezuela. Fax: 58-261-7868631.

E-mail arojasa@cantv.net 
$\mathrm{E}^{1}$ cáncer de mama es la enfermedad maligna más común en mujeres a nivel mundial ${ }^{1}$. En Venezuela, es el segundo en frecuencia en la mujer provocando alrededor de $20 \%$ de las muertes por cáncer ${ }^{2}$. Es cinco veces más frecuente en EEUU que en Japón y Taiwán y es raro antes de los 20 años, con aumento estable hasta la menopausia, seguido de un ligero incremento posterior. El riesgo aumenta con una menarquia temprana y una menopausia tardía, es más frecuente en nulípara o cuando el primer embarazo tiene lugar después de los 30 años ${ }^{3}$.

La presencia del carcinoma mamario ha ido incrementando paulatinamente, sin embargo la muerte por su causa ha ido disminuyendo, lo cual es posible atribuir al diagnóstico temprano, o al incremento de la sobrevida resultante del uso de la terapia adyuvante ${ }^{4}$. Así mismo, la evolución clínica está afectada tanto por factores pronósticos como por factores predictivos ${ }^{5-7}$. Los factores pronósticos están asociados con la aparición de metástasis tempranas o con el potencial de crecimiento del tumor primario.

Existen indicadores pronósticos que incluyen tamaño del tumor, tipo y grado de infiltración, estado de los nódulos linfáticos axilares, receptores de estrógenos, receptores de progesterona, así como también marcadores de actividad proliferati$\mathrm{va}^{7-8}$. Otros marcadores están siendo investigados por su utilidad clínica ${ }^{5}$. Mientras que los factores predictivos están asociados con la sensibilidad relativa 0 resistencia a terapias específicas ${ }^{6}$. Uno de los marcadores moleculares tanto predictivo como pronóstico lo constituye la amplificación del proto-oncogén c-erbB-2 (Her $2 / \mathrm{neu})^{9}$. Este gen se encuentra localizado en el cromosoma 17 región q21 y codifica una proteína de $185 \mathrm{Kd}$, la cual tiene actividad de tirosina quinasa.

La asociación entre amplificación del gen cerbB-2 y cáncer de mama se relacionó por primera vez en 1987, por Slamon y $\operatorname{col}^{10}$, cuyos resultados mostraron que la amplificación de este gen se relacionaba con el tiempo de recaída y sobrevida libre de enfermedad. La identificación de la sobreexpresión del oncogén c-erbB-2 se ha demostrado a través de múltiples técnicas, como el Southern blot, Spot blot, reacción en cadena de la polimerasa (PCR), inmunohistoquímica (IHC), hibridación in situ fluorescente (FISH) y más recientemente implementada la hibridación cro- mogénica in situ (CISH). Tanto el FISH como el $\mathrm{CISH}$ tienen la ventaja que permiten analizar simultáneamente morfología y sobreexpresión, las mismas no sufren por los artefactos de dilución que afectan a otros métodos para identificar amplificaciones génicas, son mucho más sensibles que los procedimientos de amplificación del ADN $^{11}$ y han mostrado tener una sensibilidad de $98 \%$ y una especificidad de $100 \%{ }^{12}$. El objetivo de este trabajo fue evaluar la amplificación del oncogén c-erbB-2 mediante FISH, relacionando los pacientes positivos por IHC y por FISH según tipo histológico, gradación histológica, presencia de ganglios linfáticos positivos y evolución de la enfermedad.

\section{Material y MÉTOdo}

Entre enero de 1998 y julio de 2002, se recibieron en la Unidad de Genética Médica de la Universidad del Zulia, 224 secciones de tejido, incluidas en parafina, de pacientes con diagnóstico anatomopatológico de carcinoma mamario, se obtuvo el consentimiento informado de todos los participantes en esta investigación, así como también la aprobación por parte del comité de ética de la Universidad del Zulia. De la totalidad de los pacientes, se seleccionaron 110 biopsias que resultaron positivas para c-erbB-2 por IHC entre 1 y 3 cruces, dependiendo de la intensidad de la coloración $^{13}$ las mismas fueron diagnosticadas anatomopatológicamente como carcinoma ductal o lobulillar invasivo, estratificados según la clasificación propuesta por Elston y Ellis ${ }^{13}$ con grados histológico entre I y III. El tamaño del tumor, la confirmación histológica y el estado de los ganglios linfáticos estuvo disponible para todos los pacientes. En ningún caso se estableció la presencia del ganglio centinela. Se identificó la amplificación de c-erbB-2 mediante técnicas de FISH. Se analizaron muestras de tejido incluido en parafina de $5 \mu$ de espesor, las mismas fueron cortadas y seleccionadas por el anatomopatólogo, donde se coloreó una de las láminas con hematoxilina y eosina para confirmar que se trataba de secciones de tejido canceroso, estas fueron desparafinadas en xilol, deshidratadas en alcohol, y secadas al aire utilizando un kit comercial para desparafinación (Vysis, USA). Posteriormente, se les realizó 
análisis de FISH utilizando la sonda PathVysion Her-2 DNA probe kit (Vysis, Inc. Downers Grove, IL. USA). Para verificar la calidad de la reacción de FISH se utilizaron láminas control para c-erbB-2, suministradas por el proveedor. Las señales para c-erbB-2 fueron visualizadas y contadas usando un microscopio de fluorescencia a través de filtros apropiados. El contaje de señales c-erbB-2 fue realizado por dos observadores independientes, cada uno identificó células tumorales y calculó la relación Her-2/CEP17, dividiendo el total de señales HER-2/neu entre el contaje total de señales CEP17. Sólo fueron consideradas aquellas que presentaron un nivel alto de amplificación $\geq 3$, según lo establecido por Tsuda y col en $2001^{14}$. A estos resultados se les aplicó la prueba de Chi cuadrado comparando los pacientes con amplificación positiva por FISH con aquellos que resultaron negativos, relacionándolos con gradación histológica, subtipo histológico, infiltración linfática y evolución de la enfermedad.

\section{Resultados}

El estudio inmunohistoquímico de los tumores mostró: 64/110 casos (58,18\%), con tres cruces, $34 / 110(30,90 \%)$ casos con dos cruces; y $12 / 110$ $(10,90 \%)$ casos con una cruz. Al relacionarlos con el análisis mediante FISH, la mayoría de los casos con 3 + amplificaron, lo cual resultó estadísticamente significativo $(\mathrm{p}<0,0001)$ Tabla 1 .

Cuando analizamos la gradación histológica de los tumores con la amplificación del gen c-erbB-2 se observó que la amplificación predominó en los grados de diferenciación histológica

Tabla 1. Amplificación del gen c-erbB-2 en carcinoma mamario. FISH / Inmunohistoquímica $1998-2003$

\begin{tabular}{|ccrc|}
\hline $\begin{array}{c}\text { Inmuno- } \\
\text { histoquímica }\end{array}$ & № casos & \multicolumn{2}{c|}{ FISH } \\
Positivo & Negativo \\
\hline $1+$ & 12 & 2 & 10 \\
$2+$ & 34 & 12 & 22 \\
$3+$ & 64 & 52 & 12 \\
Total & 110 & 66 & 44 \\
\hline
\end{tabular}

II y III; lo cual mostró tener significación estadística $(p<0,0001)$ Tabla 2.

El estudio histológico de los tumores mostró 110 casos (97\%) de carcinoma tipo ductal y 4 casos de tipo lobulillar (3\%). La amplificación mediante FISH reportó que la mayor amplificación se presentó en los casos de tipo ductal y comedocarcioma, pero esto no resultó significativo estadísticamente entre los diferentes subtipos histológicos $(\mathrm{P}=0,0048)$ Tabla 3.

En cuanto a la infiltración linfática, encontramos que aquellos casos que presentaron más de 6 ganglios positivos se correspondieron con el mayor número de casos amplificados mediante la técnica de FISH, $(\mathrm{p}<0,0001)$ Tabla 4.

A través de un seguimiento, de meses hasta 5 años, efectuado mediante consultas externas e ingresos hospitalarios, se determinó que de las 44 pacientes $(40 \%)$ que no presentaron amplifica-

Tabla 2. Amplificación del gen c-erbB-2 en carcinoma mamario. FISH / G radación H istológica 1998 - 2002

\begin{tabular}{|c|c|c|c|}
\hline \multirow{2}{*}{$\begin{array}{l}\text { Gradación } \\
\text { Histológica }\end{array}$} & \multirow[t]{2}{*}{ № $\operatorname{casos}$} & \multicolumn{2}{|c|}{ FISH } \\
\hline & & Positivo & Negativo \\
\hline I & 18 & 6 & 12 \\
\hline II & 52 & 28 & 24 \\
\hline III & 40 & 32 & 8 \\
\hline Total & 110 & 66 & 44 \\
\hline
\end{tabular}

Tabla 3. Amplificación del gen c-erbB-2 en carcinoma mamario. FISH / Subtipo Histológico 1998 - 2002

\begin{tabular}{|lrrc|}
\hline Subtipo & № casos & \multicolumn{2}{c|}{ FISH } \\
Histológico & & Positivo & Negativo \\
\hline Clásico & 50 & 36 & 14 \\
Comedo Carcinoma & 34 & 20 & 14 \\
Mucinoso & 12 & 2 & 10 \\
Papilar & 6 & 4 & 2 \\
Trabecular & 4 & 2 & 2 \\
Lobulillar & 4 & 2 & 2 \\
Total & 110 & 66 & 44 \\
\hline
\end{tabular}


Tabla 4. Amplificación del gen c-erbB-2 en
carcinoma mamario. FISH / Infiltración a ganglios linfáticos 1998 - 2002

\begin{tabular}{|lcrc|}
\hline Infiltración & \multirow{2}{*}{ No casos } & \multicolumn{2}{c|}{ FISH } \\
Linfática & & Positivo & Negativo \\
\hline Ganglios (-) & 28 & 2 & 26 \\
1 a 5 Ganglios (+) & 40 & 28 & 12 \\
6 a 10 Ganglios (+) & 32 & 28 & 4 \\
+ De 11 Ganglios (+) & 10 & 8 & 2 \\
Total & 110 & 66 & 44 \\
\hline
\end{tabular}

Tabla 5. Amplificación del gen c-erbB-2/her 2/neu en carcinoma mamario. FISH / Evolución 1998 - 2002

\begin{tabular}{|lcrc|}
\hline \multirow{2}{*}{\begin{tabular}{l}
\multirow{2}{*}{ la Enfución de } \\
la Enfermedad
\end{tabular}} & & \multicolumn{2}{c|}{ FISH } \\
& & Positivo & Negativo \\
\hline Sin Enfermedad & 38 & 8 & 30 \\
Metástasis & 36 & 26 & 10 \\
Muerte & 36 & 32 & 4 \\
Total & 110 & 66 & 44 \\
\hline
\end{tabular}

ción, cuatro (9.09\%) murieron entre los 3 y 4 años de evolución, diez (22,72\%) presentaron metástasis después de 4 años de evolución y 30 (68.18\%) permanecen en remisión entre 2 y 5 años de evolución. Con relación a las 66 pacientes que presentaron amplificación, más de $75 \%$ de los casos murieron o han presentado metástasis temprana, hecho que resultó altamente significativo $(\mathrm{p}<0,0001)$ Tabla 5.

\section{Discusión}

La creciente demanda en la identificación de la amplificación de este gen, así como también la necesidad de estandarizar resultados que sean comparables entre los laboratorios, para de esta manera referir a estos pacientes a los hospitales para un tratamiento más adecuado, constituye hoy uno de los principales retos en la obtención de un método confiable y accesible que permita identificar amplificación de este oncogén.
El análisis de nuestros datos revela que sólo $60 \%$ de los casos positivos por IHC mostraron amplificación; en los 46 casos de pacientes que presentaron entre 1 y 2 cruces por IHC sólo $50 \%$ de ellos amplificaron, mientras que de los 64 casos que presentaron 3 cruces, más de $80 \%$ amplificaron por FISH, hecho que resultó significativo estadísticamente. Se ha señalado que el HercepTest (test para IHC) no tiene una alta sensibilidad en la detección de la sobreexpresión del oncogén c-erbB-2 y que por otro lado es capaz de aportar falsos positivos ${ }^{12,15-17}$, hecho que corroboramos en este estudio.

La gradación histológica no ha sido ampliamente aceptada como factor pronóstico, por ser un método subjetivo y estar asociado a problemas de consistencia y reproductividad. Sin embargo, algunos estudios han comprobado que la gradación histológica III está asociada a ciertos marcadores IHC considerados de mal pronóstico ${ }^{13,18-20}$. Cuando comparamos la gradación histológica con amplificación o no del oncogén c-erbB-2, la casi totalidad de los casos amplificados correspondieron a los grados histológicos II y III, esto resultó estadísticamente significativo.

En cuanto a los subtipos histológicos, el tipo comedocarcinoma ha sido el mayormente relacionado con amplificación de este oncogén ${ }^{19}$, nuestros resultados no señalan una diferencia estadísticamente significativa entre los diferentes subgrupos histológicos. El significado pronóstico tradicional de factores en cáncer de mama, incluye tamaño del tumor primario, numero de ganglios axilares involucrados y el tiempo de menopausia de la paciente. A estos datos se han agregado recientemente grado nuclear e histológico, receptores de estrógeno y progesterona, porcentaje de proliferación de las células tumorales, amplificación del oncogén c-erbB-2 y estado del cathepsin $\mathrm{D}^{7,8}$. Sin embargo, la presencia de ganglios negativos permanece como uno de los más importante factores pronóstico en cáncer de mama.

En relación con la presencia o no de ganglios linfáticos infiltrados por el tumor, Simpson y col${ }^{20}$ señalaron que aquellos pacientes con ganglios axilares comprometidos, y actividad proliferativa, especialmente mitótica, estaría relacionada con una disminución de sobrevida libre de enfermedad, hecho que ha sido corroborado por otras investigaciones $^{21,22}$, por otro lado, aquellos pacientes que 
presentan ganglios linfáticos negativos y amplificación c-erbB-2 presentaron un tiempo de sobrevida más corto ${ }^{23,24}$. Sin embargo, existe un buen número de publicaciones que no muestran esta relación ${ }^{25-28}$. De las pacientes analizadas por nosotros que presentaron ganglios linfáticos negativos, dos de ellas presentaron amplificación c-erbB-2, en las mismas se observó metástasis y muerte temprana, encontrándose una mayor amplificación en los casos con más de 6 ganglios comprometidos, esto resultó estadísticamente significativo.

Winstanley y Gullick ${ }^{21}$ señalaron que el cerbB-2 actúa como un indicador pronóstico independiente en pacientes con cáncer de mama, otros han encontrado diferencias estadísticamente significativas en cuanto a la recurrencia o sobrevida de los pacientes con amplificación c-erbB$2^{10,29-31}$. La sobreexpresión de éste se ha señalado como conducente a la transformación neoplásica, como consecuencia de la actividad de la proteína Her-2/neu para promover la tumorogenicidad de otras células no malignas, debido a que la misma conduce a la transformación neoplásica de células NIH-3T3 y células de tejido mamario humanas inmortalizadas ${ }^{32-34}$. Nosotros observamos metástasis y muerte temprana en los pacientes con amplificación de este oncogén, independientemente de la presencia o no de los otros factores pronósticos analizados en este trabajo, hecho que resultó altamente significativo. Concluimos que es necesario identificar la amplificación de este oncogén como factor importante de mal pronóstico. Por otro lado, consideramos que el FISH debería utilizarse de forma rutinaria para la identificación de la amplificación de este oncogén por constituir una técnica confiable en cuanto a sus resultados; sobre todo si tomamos en cuenta que en la

\section{REFERENCIAS}

1. Greenlee RT, Murray T, Bolden S, Wingo PA. Cancer statistics 2000. Ca Cancer J Clin. 2000, 50: 7-34.

2. Ministerio Se Sanidad y Asistencia Social (Venezueia). Anuario de Epidemiologia y estadística año 1998.

3. Fremgen AM, Bland KL, Mcginnis JR. Clinical highlights from the National Cancer Database, 1,999. Ca Cancer J Clin. 49:145-58

4. Mettun C. Global breast cancer mortality statistics. Ca Cancer J Clin. 1999, 49: 138-44. actualidad se cuenta con un tratamiento específico para aquellas pacientes con amplificación de este oncogén.

Hasta el presente, existe cierta confusión entre los clínicos y patólogos en cuanto a la utilización de cualquiera de estas técnicas y sobre cuál sería el método más apropiado de usar como prueba para identificar amplificación del oncogén c-erbB-2, debido a los problemas de interpretación en las mismas ${ }^{35-37}$. Actualmente se están tratando de introducir nuevas técnicas, señaladas como de mayor sensibilidad, las mismas incluyen la hibridación cromogénica in situ $^{38}$ y la utilización de microarreglos en tejido incluido en parafina, que permite analizar varias muestras a la vez en idénticas condiciones y mejorar la calidad interpretativa del mismo ${ }^{11}$, estas constituirían una herramienta menos costosa que las técnicas de FISH. Sin embargo, estas últimas aún no han sido aprobadas su utilización por la Food and Drug Administration de los Estados Unidos de Norteamérica (FDA), ésta recientemente aprobó la utilización de dos pruebas para este propósito y de esta manera determinar la susceptibilidad al tratamiento con Trastuzumab, droga de elección en estos pacientes ${ }^{39,40}$. Las opiniones contrarias al uso del FISH en la identificación de la amplificación del cerbB-2 están basadas en los costos excesivos de esta prueba, sobre todo a nivel de países de América latina, donde se hacen en algunas oportunidades inalcanzables. Finalmente la FDA aprobó no obligar a los patólogos a usar un test particular ${ }^{41}$. Sin embargo, es necesario recalcar la importancia que tiene el FISH en la identificación de la amplificación del oncogén c-erbB-2, gracias a la susceptibilidad y confiabilidad del mismo.

5. Hayes DF, Trock B, Harris AL Assessing the clinical impact of prognostic factors: when is "statistically significant" clinically useful. Breast Cancer Res. Treat. 1998, 52: 305-19

6. Henderson IC, Pate K. The relationship between prognostic and predictive factor in the management of breast cancer. Breast Cancer Res. Treat. 1998, 52:261-88

7. Page DL, Jensen RA, Simpson JF. Routinely available indicator of prognosis in breast cancer. Breast Cancer Res. Treat. 1998, 51: 195-208 
8. Fitzgibbons PL, Page DL, Weaver D. Prognostic factor in breast cancer: College of American Pathologist consensus statement. 1999. Arch Pathol Lab Med. 2000, 124: 966-78.

9. Kaptain S, Tan L, Chen B. Her-2/neu and breast cancer. Diag Mol Pathol. 2001, 10: 139-152.

10. Slamon DJ, Ciark GM, Wong SG. Human Breast Cancer: Correlation of relapse and survival with amplification of the Her-2/neu oncogen. Science 1987, 235: 177-182.

11. Gupta D, Middieton L, Whitaker M, Abrams J. Comparison of fluorescence and Chromogenic In Situ Hybridization for detection of Her-2/neu Oncogene in Breast Cancer. Am J Clin Pathol. 2003; 119:381-387.

12. Siamon D, Godolphin W, Jones L, Holt J, Wong S, Keith D, et al. Studies of the HER-2/neu protooncogene in human breast cancer. Science 1989; 244: 707-712.

13. Thomsom T, Hayes M, Sinem J, Himand E, Sawrenko Ch, Phiwips D, Dupuis B, Parker R. Her-2/neu in breast cancer: Interobserver Variability and performance of Immunohistochemistry with 4 antibodies compared with fluorescent in situ Hybridization. Mod Pathol 2001; 14:11 1079-1086

14. ELSTON CW, Eus IO. Pathological prognostic factor in breast cancer. The value of histological grade in breast cancer: experience from a large study with long term-follow-up. Histopathology 1991, 19:403-410

15. Tsuda $H$, Akiyama $F$, Terasaki $H$, Hasegawa $T$, Kurosumi M, Shimadzu M, et al. Detection of Her2/neu(c-erb B-2) DNA amplification in Breast Cancer. Cancer 2001; 92:2965-2974

16. JacoBs TW, GRow AN, YazjI H. Comparison of fluorescent in situ hybridization and immunohistochemistry for the evaluation of Her-2/neu in breast cancer. J Clin Oncol 1999, 17: 1.974-1982.

17. Pauletti G, Dandekar S, Rong H. Testing for Her$2 /$ neu in breast cancer: Is fluorescent in situ hybridization superior in predicting outcome ?: on: assessment of methods for tissue-based detection of the Her-2/neu alterations in human breast cancer. A direct comparison of fluorescent in situ hybridization and immunohistochemistry. J Clin Oncol. 2000, 18: 3651-3654.

18. Thompson T, Hayes M, Sinelu J, Hiland E, Samrenko CH, PHILIPS D. HER-2/neu in Breast Cancer: Interobserver variability and performance of immunohistochemistry with 4 antibodies com- pared with fluorescent insitu hybridization. Moder Pathology 2001; 14: 1079-1086

19. Bцоом HJG, RichaRdson WW. Histological grading and prognosis in breast cancer. A study of 1409 cases of which 359 have been followed for 15 years. Br J Cancer 1957, II: 359-77.

20. Singletary SE, Ashiey Alfred P. Revisión of the American Joint Committee on cancer staging System for breast cancer. J Clin Oncol 2002, 20: 3628 - 36.

21. Simpson J, Gray R, Dressier L, Cobau CH, Falkson C, Gilchrist K, et al. Prognostic value of histologic grade and proliferative activity in axillary nodepositive breast cancer: Results from the eastern cooperative oncology group companion study, EST 4189. J Clin Oncol 2000, 18: 2059-2069.

22. Winstanley J. Cooke T, Murray GD. The long-term prognostic significance of c-erbB-2 in primary breast cancer. Br J Cancer 1991; 63: 447-50

23. Ross JS, Fletcher JA. The Her-2/neu oncogen. Prognostic factor, predictive factor and target for therapy. Semin Cancer Biol. 1999, 9: 125-38

24. Press MF, Bernstein L, Thomas PA. Her-2/neu gene amplification characterized by fluorescence in situ hybridization. Poor prognosis in node negative breast carcinomas. J Clin Oncol. 1997; 15: 2894-2904.

25. Borg A, BaLdetor B, Ferno M. ERB B2 Amplification predicts poor survival in node positive breast cancer. Cancer Res. 1990; 50: 4332-7

26. HaRtmann LC, Ingle JN, Woid LE. Prognostic value of c-erbB-2 overexpresion in axillary lymph node positive breast cancer. Result from a randomized adjuvant treatment protocol. Cancer 1994: 74: 2956-34

27. Noguchi M, Koyasaki N, Ohta N. C-erbB-2 oncoprotein expression versus mammary lymph node metastases as additional prognostic factors in patient with axillary lymph node-positive breast cancer. Cancer 1992; 69: 2953-60.

28. Holand JG, Barraclough BH, Gebski V, Miliken J, BıLous M. Prognostic significance of c-erbB2 oncogen in axilary node negative breast cancer. The Australian \& New Zeland J. Of Surgery 1996, 66: 64-70.

29. Andrelis IL, Bull GB, Blackstein ME. For the Toronto breast cancer study group. Neu / erb B-2 amplification identifies a poor prognosis group of women with node negative breast cancer. J Clin Oncol. 1998; 16: 1340-1349 
30. Gusterson BA, Gelber RD, Goldhirsch A. Prognostic importance of c-erb B-2 expression in breast cancer. J Clin Oncol. 1992; 10:1049-56

31. Babiak J, Hugh J, Poppema S. Significance of c-erb B2 amplification and DNA aneuploidy. Cancer 1992; 70: 770-776.

32. Borg A, Baldertorp B, Ferno M. Erb B 2 amplification in associated with high rate of proliferation. Oncogene 1991; 6: 137-43

33. Chazin VR, Kaleco M, Hiuer AD. Transformation mediated by the human Her 2/neu gene independent of the epidermal growth factor receptor. Oncogene 1992; 7: 1859-1866

34. Di FP, Pierce JH, Kraus MH. erb B-2 is a potent oncogene when over expressed in NIH/3t3 cells. Science 1987; 237:178-182

35. REESE DM, SLAMon DJ: Her-2/neu signal transduction in human breast cancer and ovarian cancer. Stem cells 1997; 15: 1-8.

36. Sauer T, Wiedswang G, Boudjema $G$, Christensen $H$, KARENSEM R. Assessment of Her-2/neu over expression and for gene amplification in breast cancer: Should in situ hybridization be the method of choice? APMIS 2003, 11:444450.

37. YAZIJI H, Gown A. Controversies and guidelines in tissue-based Her-2/neu testing in breast cancer. Med Lab Obs 2002; 34:12-6, 20-1

38. O'Grady A, Flahavan C, Kay E, Barret H, Leader M. Her-2 analysis in tissue microarrays of archival human breast cancer: Comparison of immunohistochemistry and fluorescent in situ hybridization. Applied immunohistochemitry \& Mol. Morphol. 2003, 2:177-182.

39. Ross JS, Fietcher JA. The Her 2/neu oncogene: prognostic factor, predictive factor and target for therapy. Semin Cancer Biol. 1999; 9:125-38

40. Rhodes A, Borthwick D, Sykes R, Al-Sam S, Paradiso A. The use of cell line standards to reduce her-2/ neu assay variation in multiple European cancer center and the potential of automated image analysis to provide for more accurate cut points for predicting clinical response to trastuzumab. Am J Clin Pathol. 2004, 122:51-60.

41. NELSON NJ. Experts debate value of her 2 testing methods. J Nat Cancer Inst. 2000; 92: 292 - 294. 